\title{
Pragmatic approach to provide uninterrupted obstetric services amidst SARS COV-2 pandemic
}

\begin{abstract}
The novel severe acute respiratory syndrome corona virus 2 (SARS COV-2) has taken our civilization by surprise. Eachpassing day emanate new set of issues, necessitating changes in its management. Its significance \& impact on pregnant woman, pregnancy outcome has been detrimental as COVID-19 has been associated with maternal and perinatal morbidity and mortality in this set of vulnerable groups-pregnant women. The recommendations by ICMR need to be appreciated and efforts should be made at each level to simplify the assessment of COVID-19. We at a tertiary private setup followed methodology for triage and assessment for pregnant women ever since lock down measures was levied. The present paper discusses in brief the approach that was followed at our institute that helped us to provide services without fail during the crisis hour as well as minimizing the risk of infection to our doctors, resident doctors, nursing staff, support staff and health care workers in the team. This could provide valuable inputs to other institutes facing dearth of skilled man power as we all need to gear up for coming months as the pandemic is ongoing with no flattening of curve in near sight.
\end{abstract}

Volume 6 Issue 6 - 2020

\section{Brinderjeet Kaur, S Fayyaz}

Department of Obstetrics and Gynecology, SantokbaDurlabhji Memorial Hospital and Research Center, India

Correspondence: Brinderjeet Kaur, Consultant, Department of Obstetrics and Gynecology, SantokbaDurlabhji Memorial Hospital and Research Center, Jaipur, India,

Email dr.jkaur@gmail.com

Received: October 21, 2020 | Published: November 19, 2020

Keywords: obstetrics, SARS COV-2 pandemic, pregnancy

Abbreviations: CLIA, chemiluminescent immunoassay; ELISA, enzyme linked immunosorbent assay; LFA, lateral flow assay

\section{Introduction}

The novel severe acute respiratory syndrome corona virus 2 (SARS COV-2) is the virus responsible for COVID-19, that has taken our civilization by surprise. ${ }^{1}$ The mystery behind its behavior is still unraveled. Each passing day emanate new set of issues, necessitating changes in its management. It's significance \& impact on pregnant woman, pregnancy outcome has been detrimental as COVID-19 has been associated with maternal and perinatal morbidity ${ }^{1,2}$ and mortality in this set of vulnerable groups-pregnant women. The escalating number of patients in early phase of pandemic shocked medical fraternity world over and entire focus was on establishment of intensive care units, lockdown, getting acclimatized to new social norms-social distancing and use of masks.

The impact on pregnant women took backseat as evidenced by initial ICMR guidelines that stated that pregnant women seem to be less likely to be infected with COVID-19 in comparison the general population. A recent study by ICMR-NIRRH in the state of Maharashtra ${ }^{3}$ highlights that $12 \%$ of the pregnant women are affected by COVID-19 infection, of which $88 \%$ are asymptomatic. This is clear reflection that we initially recognized the tip of iceberg considering high fertility rate in India, if we extrapolate the incidence of COVID-19 infection in pregnant women the numbers could be alarming. It therefore mandated ICMR to propose strategies for management of pregnant women approaching delivery and revision in advisory as well as new set recommendations. ${ }^{4}$ under:

The salient features of new recommendations are enumerated as

a. All pregnant women residing in cluster or containment areas from hotspot districts who present with signs of labor or are likely to deliver in the next five days should be tested for COVID-19 even if asymptomatic.

b. Asymptomatic direct and high -risk contacts of a confirmed case are to test once between 5 and 1 days of coming in his/her contact.

c. Hospitals to maintain a registry for all women admitted with confirmed COVID-19 infections in pregnancy.

d. Pregnant women with heart disease (congenital or acquired), to be considered as high-risk group.

The recommendations by ICMR need to be appreciated and efforts should be made at each level to simplify the assessment of COVID-19. We at a tertiary private setup in Jaipur followed methodology for triage and assessment for pregnant women ever since lock down measures was levied. The present paper discusses in brief the approach that was followed at our institute that helped us to provide services without fail during the crisis hour as well as minimizing the risk of infection to our doctors, resident doctors, nursing staff, support staff and health care workers in the team. This could provide valuable inputs to other institutes facing dearth of skilled man power as we all need to gear up for coming months as the pandemic is ongoing with no flattening of curve in near sight.

\section{Testing}

The diagnostic tests for SARS-COV2 Virus are of three types. ${ }^{5,6}$

I. Molecular Assay-Reverse Transcriptase Polymerase Chain reaction (RT-PCR), Nucleic amplification test (NAT)-The RTPCR based test is recommended gold standard test for diagnosis of SARS-COV2 infection. They require nasopharyngeal or oropharyngeal swab, but the test is accurate although it requires expertise and is time consuming. 
II. Serological tests- Rapid antibody tests- The serology-based tests require blood (venous or finger prick), are quick, require minimum expertise and are able to diagnose past SARSCOV2 infection. The tests utilize following antigens of SARSCOV2:Spike (S) protein - Surface protein, Nucleocapsid (N) protein andReceptor binding domain (RBD). ${ }^{2}$

The serology test can be done by different techniques ${ }^{7,8}$

a. Neutralization assay

b. Chemiluminescent immunoassay (CLIA)

c. Enzyme linked immunosorbent assay (ELISA)

d. Lateral flow assay (LFA)-The common card type

e. The propensity of cross reactivity, inability to detect acute / recent infection, lack of an immunity marker status was some of the fallacies that led CDC and FDA to abandon this antibody test. The ICMR also didn't approve these tests except of emergent point of care use

\section{Application of serology test principles in practice}

Women who do not know their infectious status represent a potential risk not only for themselves, for the fetus but also to the health care workers and other patients in her vicinity. Some state governments in India have suggested that the detection of antibody status to SAR-COV -2 could serve as a basis for" immunity passport "or "risk -free Certificate "even if the supposed immunity is short lived. Testing pregnant women for antibody response to COVID-19 may have different advantages, such as identifying

a. Possibly women who have overcome the dreaded disease "healed"women with Ig G positive who were never tested with real -time reverse transcription polymerase chain reaction (RTPCR)

b. Women who are at risk for COVID-19 infection i.e both IgM and Ig G negative.

c. Third set which are in infective state - having IgM positive and Ig G negative

d. An interesting set with Both Ig M and Ig G positive.

There are different ways to test for antibody against SARS-COV2. The most commonly used method $\mathrm{s}$ are IgM and IgG titer measured by either chemiluminescence immunoassay analysis or enzymelinked immunosorbent assay and a rapid (result within 15 minutes) IgM-IgG combined antibody test. The sensitivity and specificity of these antibody test are still being studied and at present sensitivity rate reported to be $48 \%, 89 \%, 89 \%$ respectively and specificity rate $100 \%, 91 \%$ and $91 \%$ respectively. ${ }^{9-11}$

The use of this rapid combined antibody test can be used as a point -of- care of paramount importance especially in obstetrics health care setting. The interpretation of rapid test could give us insight to status of pregnant woman immune response to COVID-19 infection with different sets of permutations and combinations of two commonly used antibodies, IgM \& IgG.
a. IgG negative and $\operatorname{IgM}$ negative (Susceptible to infection)
b. IgG positive and IgM negative (Healed phase)
c. IgG positive, IgM positive (Possibly infective)
d. IgG negative and IgM positive (Infective state)

e. The presence of IgM increases the possibility that the tested person is still contagious. ${ }^{12}$

\section{Antigen based rapid test $t^{7,8}$}

The antigen-based assay affords rapid active SARS-COV2 infection using nasal or throat swabs within an hour. As the sample requirement is same as that of RT-PCR, the preanalytical variables like collection technique, transport play vital role affecting the results. These tests have high chances of missing an active infection and a negative test needs to be confirmed by molecular test.

\section{Algorithm of care in pregnant women}

At our setup, all pregnant women nearing delivery are tested with RT PCR and rapid serology \& or antigen test before hospital admission. The rapid test results are available in 15-30 minutes. This is of immense help as it gives an initial insight into patient's COVID status. If the rapid test is positive to SARS-COV-2, the women are considered as COVID-19 positive with RTPCR done. The time until RT PCR test result are available the woman is kept in isolation. If it is possible to postpone the intervention as in cases of planned cesarean delivery, induction of labor or any other elective surgical procedure the patient is counselled, intervention postponed to later date and is referred for COVID unit for necessary work up for COVID, treatment if at all required. It is extremely important that we do not rush away with elective procedures without ruling out COVID infection by RT PCR test-the gold standard of diagnosis.

Waiting for both the serology and more confirmatory RTPCR report makes sense in elective or non-emergency cases as it:

I. Allays anxieties among the health care workers (HCW) about COVID-19 status of the patient.

II. Overcomes the potential shortage of personal protective equipment by avoiding use of PPE in non COVID patient.

III. Imposes less burden on the health care providers, by limiting their hours of work and causing undue burden on the work force of hospital.

IV. Decreases the chances of potential complications of the patient being subjected to procedure while being affected with COVID -19 .

V. Helps in better utilization of resources like isolation rooms or oxygen supplies, ventilators and ICU facilities.

If it is not feasible to postpone the admission (eg, laboring or bleeding pregnant women), the patient is admitted to the COVID unit and managed as COVID-19 positive (Figure 1). A separate area has been allocated for such cases. The COVID area is specially cordoned off area or isolation room with negative pressure. It has facilities of 2 laboring tables with all requirements of conducting a normal delivery and OT table with arrangements for caesarean or operative procedure with due working environment for anesthetist and baby resuscitation trolley. Patients are managed by experienced obstetricians with third level of protective equipment. After every procedure the delivery room undergoes mandatory fogging and sanitization with hypochlorite. Patients are recommended to wear surgical masks. the access to the family members of the patient is not allowed and one healthy attendant with the patient is only allowed (Figure 1). After the procedure the patient is shifted to isolation area till RTPCR results are available. A negative RTPCR facilitates the shifting the patient to normal ward, while a positive RT PCR report will mandate patient to stay in isolation. 


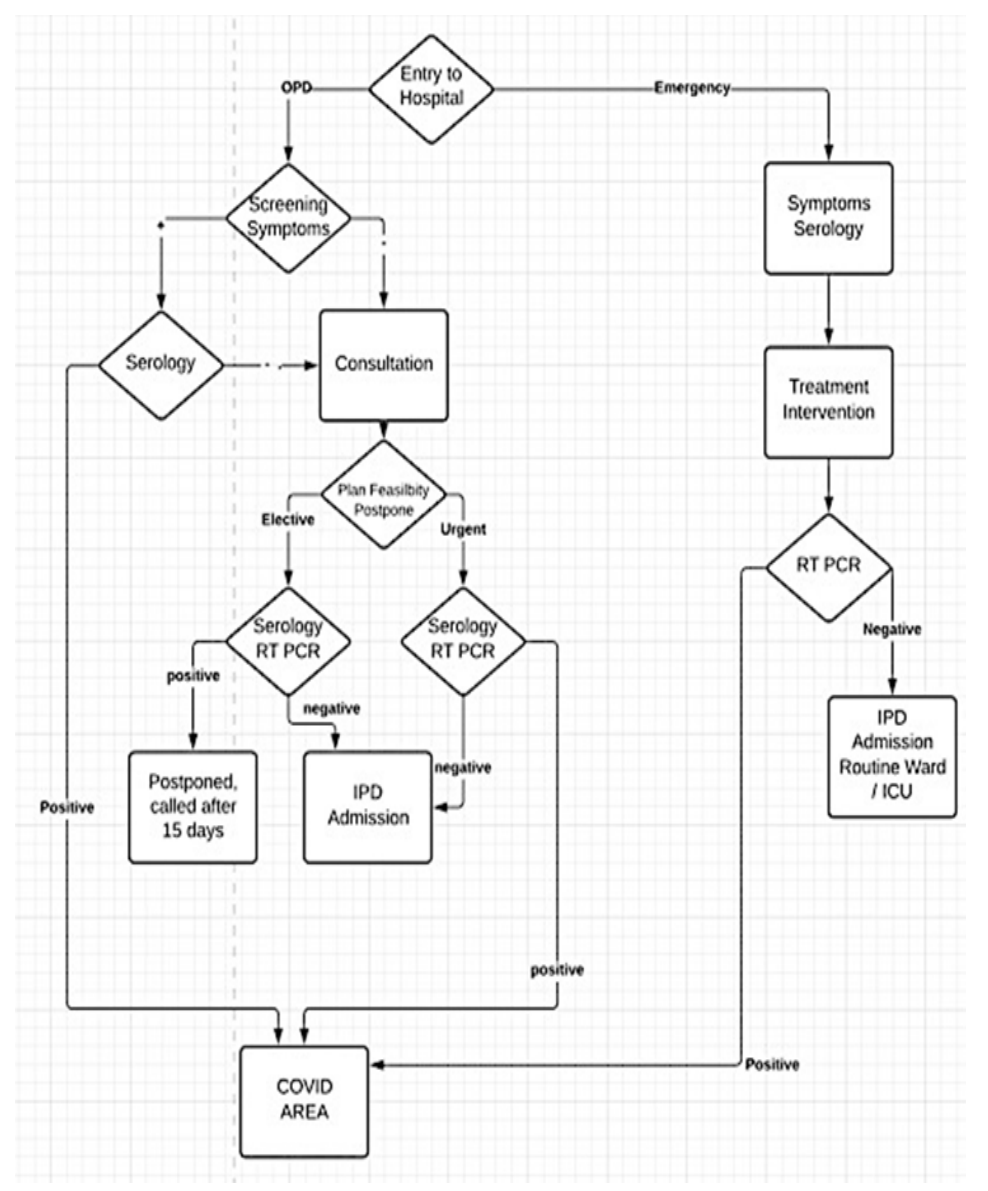

Figure I Algorithm of point of care of pregnant women on reception to our department in hospital.

In case where the serology is reported negative, in elective cases we wait for the RTPCR report. In emergency situations e.g patient is in active labor or having bleeding, it is not possible to wait for RTPCR. In such situations too the case is still deemed as positive and subjected to same protocols as in COVID-19 positive patients. However, for such cases an intermediate area has been created with 1 delivery table and all facilities of COVID positive suite. This intermediate area, prevents the chances of transmission of infection from a proven case of COVID-19 in the COVID delivery rooms. When the RT PCR report comes negative, the patient shifted to normal ward. In case, the RTPCR comes positive, the patient is kept in isolation/COVID ward. The benefit of this protocol is

a. The laxity in the approach to management is avoided.

b. Infuses confidence in the health care workers and allays the anxiety of not taking protective measures while the more confirmatory RTPCR report is awaited.

c. Helps in clearly streamlining the protocol without any area of doubt

d. If the serology and RTPCR reports are negative then the patient is admitted in normal course and with due precautions subjected to the desired procedure. The management of non-COVID patient in normal delivery rooms allays anxiety of both patient and treating team.
It would also be helpful to test visitors and HCWs, if feasible. In our department, we have mandatory rapid antigen testing for all HCWs every 7 days. HCWs that have a positive result for antigen self-isolate at home while awaiting the results of the NP swab.

It is pertinent to mention that the streamlined approach that we adopted after lock down enabled us to provide continuous obstetric care services with minimum number of our team members getting down with COVID infection. Every institute/ hospital / service provider should have written policy for handling and triage of patients specially in obstetrics and gynecology. The abidance to such policies is beneficial to all as there is no confusion in handling of patients, more confidence among $\mathrm{HCW}$ enabling better interaction \& patient care, judicious utilization of personal protective equipment which are already in shortage globally and unbridled as well as unhampered management of the department catering to the needs of both COVID and Non COVID patients without an evidence of crisis or forced shut down of the department .

\section{Creating special delivery rooms for COVID patients}

A brief mention of how we were able to create separate special delivery rooms for COVID positive patients. A set of three rooms were isolated for use as labor delivery rooms during COVID pandemic. Each room was approximately 10 X10 feet with proper ventilation with exhaust fans to create negative pressure and an attached wash room for laboring patients. The rooms were well equipped with 
laboring table, stirrups, oxygen, CTG machine, monitors and suction point. There was a small area cordoned off to contain consumables, medicines, injectables used during delivery. A baby resuscitation trolley was kept which can cater to all the three delivery rooms. One delivery room was equipped with Operation table and equipment \& monitors used by anesthetist. A small area for donning and doffing for $\mathrm{HCW}$ to be present. The patient or HCW from one delivery room were not allowed to interact with the adjoining delivery room. A common working station with facility to communicate on phone or internet was provided. Paper work was kept to minimal to bare legal requirement with no unnecessary frisking.

\section{Recommendation}

There is no glimmer of hope for end of this pandemic and it is essential that we provide uninterrupted obstetric services which could only be achieved if we are able to maintain our front-line health care work force intact \& free of COVID-19 infection. We suggest mandatory point of care testing strategy for SARS-CoV-2 for pregnant women before receiving care in settings, as feasible. Those who have a positive result should undergo a NP swab and admission or appointment should be postponed, if feasible, until NP swab test results are available till thenthey should be considered COVID-19 positive.

\section{Acknowledgments}

None.

\section{Conflicts of interest}

Author declare that there is no conflict of interest.

\section{Funding}

None.

\section{References}

1. Pierce-Williams RAM, Burd J, Felder L, et al. Clinical course of severe and critical COVID-19 in hospitalized pregnancies: a US cohort study. Am J Obstet Gynecol MFM. 2020;2(3):100134.

2. Breslin N, Baptiste C, Gyamfi-Bannerman C, et al. COVID-19 infection among asymptomatic and symptomatic pregnant women: two weeks of confirmed presentations to an affiliated pair of New York City hospitals. Am J Obstet Gynecol MFM. 2020;2(2):100118.

3. Rakesh Waghmare, Rahul Gajhiye, Niraj Mahajan, et al. Universal screening identifies asymptomatic carriers of SARS-CoV -2 among pregnant women in India. European Journal of Obstetrics \& Gynecology and Reproductive Biology. 2020.

4. Guidelines for Management of Pregnant women in COVID.

5. John Hopkins university and medicine.

6. Coronavirus Disease 2019 Testing Basics.

7. Molecular based Test.

8. STANDARD Q COVID-19 Ag.

9. Sethuraman N, Jeremiah SS, Ryo A. Interpreting diagnostic tests for SARS-CoV-2. JAMA. 2020.

10. Jin Y, Wang M, Zuo Z, et al. Diagnostic value and dynamic variance of serum antibody in coronavirus disease 2019. Int J Infect Dis. 2020;94:4952.

11. Li Z, Yi Y, Luo X, et al. Development and clinical application of a rapid IgM-IgG combined antibody test for SARS-CoV-2 infection diagnosis. J Med Virol. 2020;25727.

12. Hall MA, Studdert DM. Privileges and immunity certification during the COVID-19 pandemic. JAMA. 2020;323(22):2243-2244. 